\title{
Regeneración posincendio de plantas leñosas en plantaciones de Pinus radiata D. Don, zona costera, Región del Maule, Chile central
}

\author{
Post-fire regeneration of woody species in Pinus radiata D. Don plantations, Coastal \\ zone, Maule Region, central Chile
}

\author{
Persy Gómez* \& Steffen Hahn \\ Jardín Botánico, Universidad de Talca, Casilla 747, Talca, Chile. \\ *pegomez@utalca.cl
}

\begin{abstract}
We evaluated the regeneration of woody species in Pinus radiata plantations. The objective was to determine the recovery of vegetation from fires. The results indicated that $93 \%$ of the species are resprouters already present in the site before the fires. The most representattive familia were Anacardiaceae, Fabaceae and Rosaceae. Considering frecuency, coverage and relative density, the most important species are: Lithrea caustica, Schinus polygamus and Kageneckia oblonga. Lithrea caustica was the species that generated a great amount of sprouts some of them reaching to $95 \mathrm{~cm}$. There was no regeneration of $P$. radiata but it may be only a matter of time.
\end{abstract}

El fuego es una perturbación que a nivel global afecta las comunidades y al paisaje vegetal (Bond \& Van Wilgen 1996). Análisis de la dinámica de bosques naturales, plantaciones de coníferas y cambios en el paisaje producto de incendios han sido desarrollados entre otros por Oliver \& Larson (1997), Quintanilla (2000), Fuller (2001), González \& Bravo (2001), Fulé et al. (2003), Keeley et al. (2003) y Castillo (2006).

En ecosistemas mediterráneos el fuego es un factor natural con influencia en la productividad biológica y composición vegetal (Iniguez et al. 2008). Postincendio $\mathrm{y}$ tras el deterioro y pérdida de vegetación se generan espacios abiertos donde se activa la germinación del banco de semillas, así como el rebrote de individuos de especies pre-existentes (Calvo et al. 2008, Weiguo et al. 2008, Vidal \& Reif 2011). Además especies pioneras o invasoras son favorecidas al colonizar sitios perturbados (Pickett et al. 1987, Richardson et al. 1994, Cóbar-Carranza et al. 2015).

Para la zona central de Chile las condiciones de estacionalidad han estimulado eventos de fuego con cuantiosas pérdidas ambientales, sociales y económicas (Castillo et al. 2012). Para el caso de plantaciones con especies exóticas, desde los años 90 se ha observado un aumento en incendios, con tendencia creciente en frecuencia y superficie afectada por año (González et al. 2011). Por otra parte, para el siglo XX respecto a períodos previos, estudios dendroclimáticos dan cuenta de un incremento significativo en recurrencia de sequías y disminución de precipitaciones (Le Quesne et al. 2006).
En ausencia del fuego, y en rodales juveniles de Pinus radiata $\mathrm{D}$. Don de primera o segunda rotación, del centro sur de Chile, se desarrolla bajo el dosel un matorral con especies originales del sitio (Ramírez et al. 1984, Gómez et al. 2009). Sin embargo, tras la ocurrencia de incendios, ocurren cambios en la composición florística de estos matorrales, sobreviviendo especies leñosas capaces de resistir y rebrotar, a causa de la presencia de tallos, raíces y/o lignotúberes (Montenegro et al. 1983, Ginocchio et al. 1994, Figueroa \& Jacksic 2004, Montenegro et al. 2004).

En este marco de posincendios y en plantaciones de $P$. radiata es relevante pesquisar desde el punto de vista florístico la dinámica de regeneración de la vegetación leñosa y conocer cuál es la composición resultante de estos ecosistemas artificiales (Alanís et al. 2011, 2012). Los objetivos de este trabajo fueron a) confeccionar un catálogo florístico de las especies vegetales con capacidad regenerativa; b) cuantificar la distribución horizontal a través de la abundancia, dominancia, frecuencia y densidad y c) analizar estructura de tamaño de los rebrotes, estrategia regenerativa y origen biogeográfico de las especies.

El estudio se desarrolló el año 2015, en época primaveral y específicamente entre los sectores de Nirivilo y Pichamán accesible por la carretera que une la ciudad de Talca con el balneario de Constitución, Región del Maule (Fig. 1). Posterior a seis meses de ocurrido el incendio, la información se levantó de rodales de $P$. radiata, homogéneos, manejados, insertos en sitios con pendientes suaves y abundante sotobosque. En cada rodal, de aprox. 
2 ha de superficie, se realizaron 15 parcelas rectangulares dirigidas de $200 \mathrm{~m}^{2}(20 * 10 \mathrm{~m})$. El inventario se focalizó en especies leñosas determinando su abundancia, de acuerdo con el número de individuos, dominancia en función de la cobertura según porcentaje de superficie ocupada, y frecuencia absoluta. Para la densidad se consideró un individuo como cada cepa con el conjunto de rebrotes. Esta información fue complementada con datos respecto de la altura de los rebrotes, su estrategia de regeneración y estatus de las especies (Zuloaga et al. 2008).

Taxonómicamente la flora que regenera incluye 12 familias, 14 géneros y 15 especies. Las familias con mayor representatividad fueron Anacardiaceae, Fabaceae y Rosaceae con dos especies cada una. De las 15 especies registradas, 12 presentaron como estrategia regenerativa rebrotes, dos estrategias mixtas (rebrote y semillas) y una especie exhibió exclusivamente una estrategia sexual (Tabla 1).

En frecuencia, cobertura y densidad relativa, las especies con los mayores valores fueron Lithrea caustica (Molina) Hook. et Arn., Schinus polygamus (Cav.) Cabrera y Kageneckia oblonga Ruiz et Pav. (Tabla 1). Estos resultados coinciden con lo reportado por Araya \& Ávila (1981) y Montenegro et al. (1983), donde luego de un incendio, las tendencias de recuperación de las especies tanto en volumen y cobertura siguen en general patrones de crecimiento acelerado los primeros años. Además, estas respuestas, estarían ligadas a la morfología de estas plantas, adaptadas a sobrevivir frente a intenso estrés hídrico, típico de zonas con climas mediterráneos (Money \& Kummerow 1971), favoreciendo su resistencia al fuego y rápido rebrote.

Respecto del estatus de las especies, dominan ejemplares nativos por sobre los exóticos, y donde dentro de estos últimos destaca, en términos de densidad, Acacia dealbata Link (Tabla 1). Desde el punto de vista de la estructura de tamaños en altura de crecimiento, se observa que la mayor cantidad de rebrotes oscila entre 31 y $50 \mathrm{~cm}$ de altura. Lithrea caustica (litre) es la especie con rebrotes de mayor altura alcanzando $95 \mathrm{~cm}$. En contraste, los menores valores fueron de Ribes punctatum Ruiz et Pav., alcanzando solo hasta $10 \mathrm{~cm}$ (Fig. 2). Entre los diferentes taxones, 20 fue la mayor cantidad de rebrotes por cepa y observados en L. caustica. La mejor respuesta regenerativa de "litre" refleja su condición de origen mediterráneo donde además del fuego soporta largos periodos de sequía. Por otro lado, como estrategia de recuperación de la biomasa aérea, L. caustica, así como otras especies mediterráneas, desarrolla lignotúberes, protuberancias leñosas, que además de almacenar yemas presentan reservas alimenticias que pueden contribuir a proveer de energía a las plantas, cuando estas han sufrido importantes daños producto de la acción del fuego (Montenegro et al. 1983).

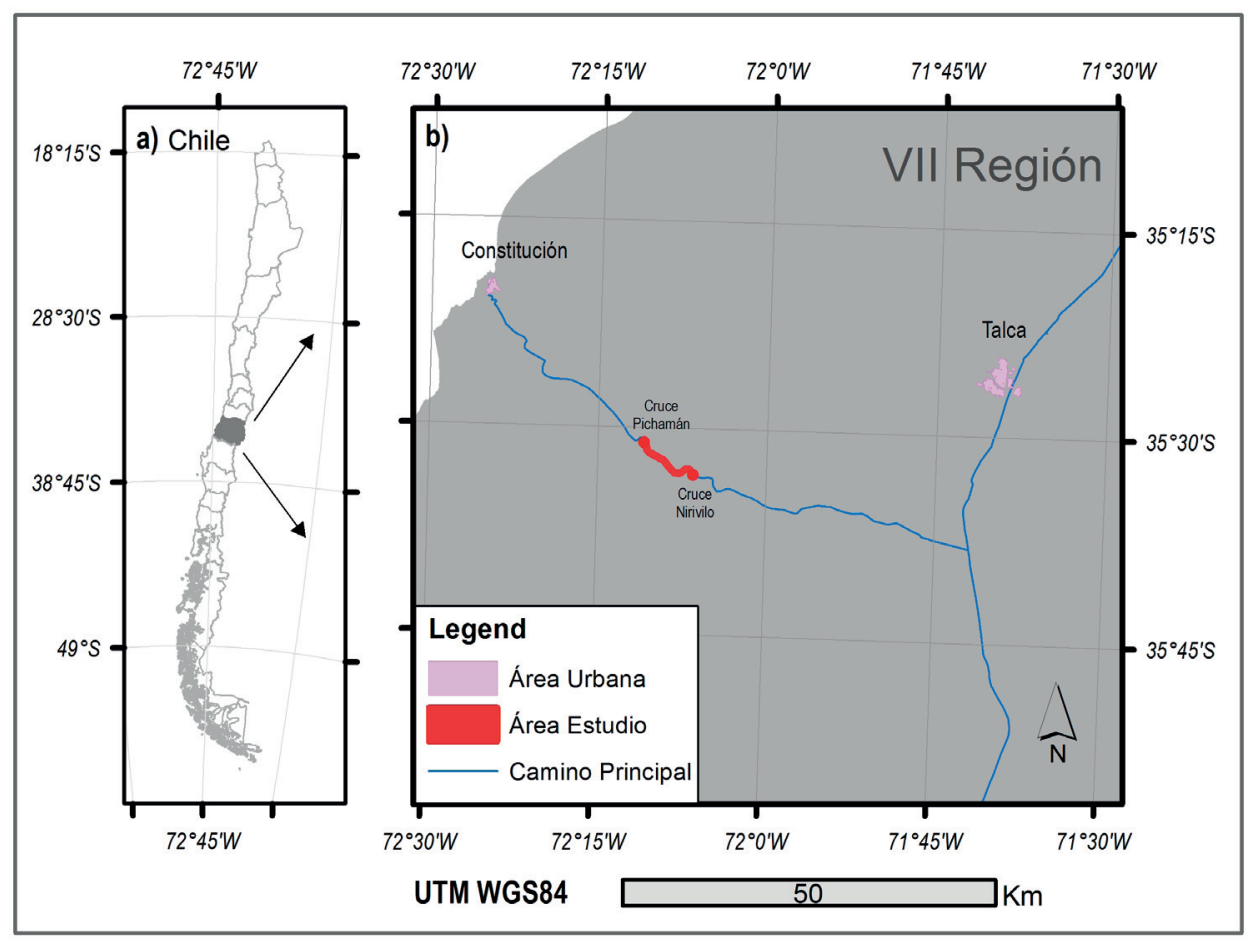

Figura 1. (a) Mapa de Chile caracterizando la Región del Maule. (b) Acercamiento área de estudio, camino a la ciudad de Constitución, entre Nirivilo y Pichamán, provincia de Talca, Región del Maule. / (a) Map of Chile characterizing the Maule Region. (b) Close-up study area, way to Constitution city, among Nirivilo and Pichaman, Talca province, Maule Region. 


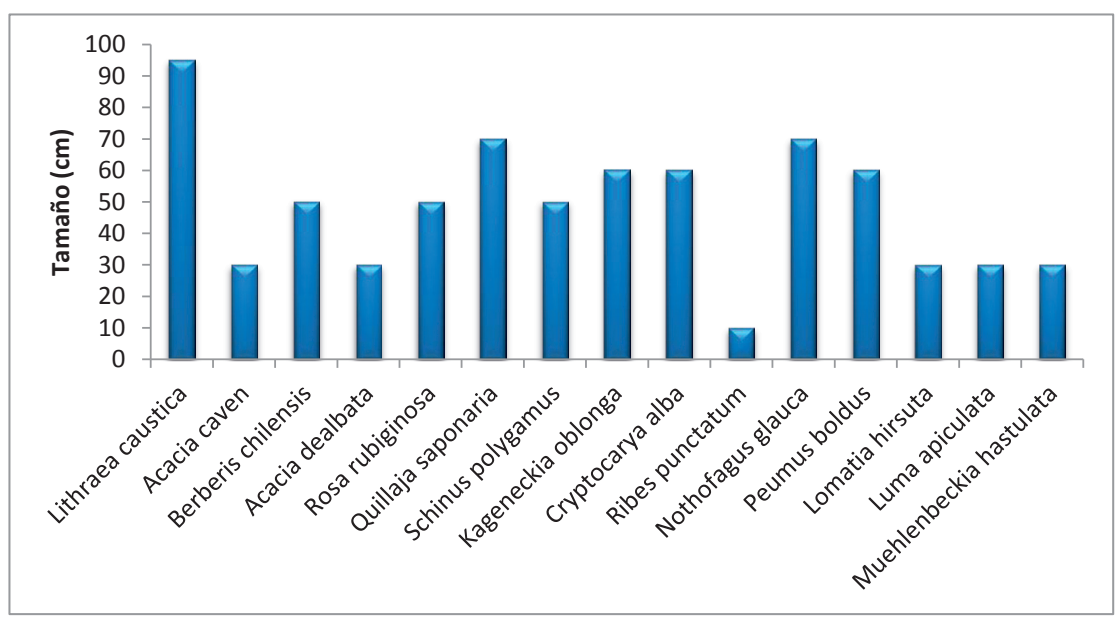

Figura 2. Alturas máximas de los rebrotes o plántulas para 15 especies leñosas, provincia de Talca, Región del Maule, Chile central. / Maximum height of the sprouts or seedlings to 15 woody species, Talca province, Maule Region, central Chile.

TABLa 1. Nombre científico, familia, estatus, estrategia reproductiva, frecuencia $(\% \mathrm{~F})$, cobertura $(\% \mathrm{C})$, abundancia total (At) y densidad relativa $(\% \mathrm{D})$ de las especies del sitio de estudio. / Scientific names, families, status, reproductive strategy, frequency $(\% \mathrm{~F})$, coverage $(\% \mathrm{C})$, total abundance $(\mathrm{At})$ and relative density $(\% \mathrm{D})$ species of the study site.

\begin{tabular}{|c|c|c|c|c|c|c|c|}
\hline ESPECIE & FAMILIA & Status & $\begin{array}{c}\text { ESTRATEGIA } \\
\text { REPRODUCTIVA }\end{array}$ & $\% \mathrm{~F}$ & $\% \mathrm{C}$ & $\begin{array}{c}\mathrm{AT} \\
\left(3000 \mathrm{~m}^{2}\right)\end{array}$ & $\% \mathrm{D}$ \\
\hline Lithrea caustica (Molina) Hook. \& Arn. & Anacardiaceae & Nativa & Vegetativa & 100 & 13 & 154 & 43 \\
\hline Acacia caven (Molina) Molina & Fabaceae & Nativa & Vegetativa & 20 & 0,5 & 3 & 0,84 \\
\hline Berberis chilensis Gillies ex Hook. \& Arn. & Berberidaceae & Nativa & Vegetativa & 6 & 0,1 & 1 & 0,28 \\
\hline Acacia dealbata Link & Fabaceae & Exótica & Vegetativa-Sexual & 20 & 1 & 14 & 3,9 \\
\hline Rosa rubiginosa $\mathrm{L}$. & Rosaceae & Exótica & Vegetativa & 6 & 0,1 & 1 & 0,28 \\
\hline Quillaja saponaria Molina & Quillajaceae & Nativa & Vegetativa & 27 & 2 & 21 & 5,87 \\
\hline Schinus polygamus (Cav.) Cabrera & Anacardiaceae & Nativa & Vegetativa & 67 & 9 & 89 & 24,86 \\
\hline Kageneckia oblonga Ruiz \& Pav. & Rosaceae & Nativa & Vegetativa & 60 & 7 & 43 & 12,01 \\
\hline Cryptocarya alba (Molina) Looser & Lauraceae & Nativa & Vegetativa & 6 & 0,1 & 1 & 0,28 \\
\hline Ribes punctatum Ruiz \& Pav. & Grossulariaceae & Nativa & Sexual & 6 & 0,2 & 12 & 3,35 \\
\hline Nothofagus glauca (Phil.) Krasser & Nothofagaceae & Nativa & Vegetativa & 33 & 3 & 12 & 3,35 \\
\hline Peumus boldus Molina & Monimiaceae & Nativa & Vegetativa & 6 & 0,1 & 1 & 0,28 \\
\hline Lomatia hirsuta (Lam.) Diels & Proteaceae & Nativa & Vegetativa-Sexual & 6 & 0,1 & 3 & 0,85 \\
\hline Luma apiculata (DC.) Burret & Myrtaceae & Nativa & Vegetativa & 6 & 0,2 & 2 & 0,57 \\
\hline Muehlenbeckia hastulata (Sm.) I.M. Johnst. & Polygonaceae & Nativa & Vegetativa & 6 & 0,1 & 1 & 0,28 \\
\hline Total & & & & & & 358 & 100 \\
\hline
\end{tabular}

De estos resultados se resalta la capacidad de rebrote de diferentes especies. Sin embargo, lo anterior, es heterogéneo, destacándose L. caustica por su mayor respuesta respecto a otras del mismo sitio. Cabe recalcar que las especies que rebrotan presentan una ventaja frente a otras no rebrotadoras, en relación con la monopolización y uso de recursos en las primeras etapas posincendio (Alanís et al. 2012).
A nivel de paisaje y en el sitio de estudio, se puede observar un rápido recubrimiento de áreas quemadas con material vegetal, lo que permite otorgar al suelo mayor estabilidad, y al mismo tiempo, crea una cobertura de protección para el crecimiento de plantas de menor envergadura. Sin embargo, las altas pendientes en algunos sectores (sobre 50\%), indican un alto grado de daño al 
suelo en casi todo el perfil, encontrándose sectores con escasa o nula vegetación. Es destacable mencionar, que las principales especies leñosas que regeneran posincendio en rodales de pino aledaños no evaluados son las mismas que se pueden observar en el sitio de nuestra investigación.

Por otra parte, en los rodales evaluados es notable la ausencia de plántulas de $P$. radiata, ya que habitualmente especies de este género no presentan problemas en regenerar posterior a un incendio (Richardson et al. 2000). Sin embargo, el no encontrar reclutas de $P$. radiata puede ser producto de la alta severidad del incendio (Alanís et al. 2008), o a la época de muestreo, puesto que la especie no madura los conos hasta el verano u otoño del año siguiente al de la fecundación (McDonald \& Laacke 1990).

Se concluye que la composición florística del sitio posincendio es diversa, con elementos de distintos estatus y pertenencia a comunidades vegetales. El 93\% de las especies rebrotadoras siguen una estrategia regenerativa vegetativa. Para la zona de estudio, y considerando cobertura, frecuencia y densidad relativa, L. caustica se presenta como la especie más importante en rodales de $P$. radiata incendiados. Se recomienda realizar nuevos estudios que evalúen a largo plazo la regeneración de plantas nativas post-incendio en plantaciones de pinos. Además, estas investigaciones debieran considerar analizar conceptos como carga de combustible, intensidad y severidad del incendio y su relación con la regeneración.

\section{AGRADECIMIENTOS}

Al Jardín Botánico de la Universidad de Talca. Al gran aporte del Prof. Jóse San Martín, Instituto de Ciencias Biológicas, y a Yony Ormazábal del Centro de Geomática de la Universidad de Talca. Además a los revisores anónimos que con sus aportes permitieron enriquecer el manuscrito.

\section{REFERENCIAS}

Alanís, E., Jiménez, J., Espinoza, D., GonzÁlez, M.A., Jurado, E., Aguirre, O.A. 2008. Monitoreo del estrato arbóreo en un área restaurada post-incendio en el Parque Ecológico Chipinque, México. Revista Chapingo serie Ciencias Forestales y del Ambiente 14: 113-118.

Alanís, E., Jiménez, J., Valdecantos, A., Pando, M., Aguirre, O., Treviño, E.J. 2011. Caracterización de regeneración leñosa post-incendio de un ecosistema templado del Parque Ecológico Chipinque, México. Revista Chapingo. Serie Ciencias Forestales y del Ambiente 17: 31-39.

Alanís, E., Jiménez-Pérez, J., Valdecantos-Lema, A., GonzÁlez Tagle, M.A., Aguirre-Calderón, O.A., Treviño-Garza, E.J. 2012. Composición y diversidad de la regeneración natural en comunidades de Pinus-Quercus sometidas a una alta recurrencia de incendios en el noreste de México. Revista Mexicana de Biodiversidad 83: 1208-1214.
Araya, S., Ávila, G. 1981. Rebrote de arbustos afectados por el fuego en el matorral chileno. Anales Museo Historia Natural, Valparaíso. 14: 107-113.

Bond, W., Van WiLgen, B.W. 1996. Fire and plants. Chapman \& Hall. 263 pp.

Calvo, L., Santalla, S., Valbuena, L., Marcos, E., Tárrega, R., Luis-Calabuig, E. 2008. Post-fire natural regeneration of a Pinus pinaster forest in NW Spain. Plant Ecology 197: 81-90.

Castillo, M. 2006. El cambio del paisaje vegetal afectado por incendios en la zona mediterránea costera de la Quinta Región de Chile. Tesis. Universidad de Chile. Santiago, Chile. 143 pp.

Castillo, M., Garfias, R., Julio, G., González, L. 2012. Análisis de grandes incendios forestales en la vegetación nativa de Chile. Interciencia 37(11): 796-804.

Cóbar-Carranza, A.J., García, R., Pauchard, A., Peña, E. 2015. Efecto de la alta temperatura de germinación y supervivencia de semillas de la especie invasora Pinus contorta y dos especies nativas del sur de Chile. Bosque 36(1): 53-60.

FigueroA, J., JACKSIC, F. 2004. Latencia y banco de semillas en plantas de la Región Mediterránea de Chile. Revista Chilena de Historia Natural 77: 201-215.

Fulé, P., Crouse, J., Heinlein, T., Moore, M., Covington, W., Verkamp, G. 2003. Mixed-severity fire regime in a high-elevation forest of Grand Canyon, Arizona, USA. Landscape Ecology 18: 465-486.

Fuller, D. 2001. Forest fragmentation in Loudon Country, Virginia (USA), evaluated with multitemporal Landsat imagery. Landscape Ecology 16: 627-642.

Ginocchio, R., Holmgren, M., Montenegro, G. 1994. Effect of fire on plant architecture in Chilean shrubs. Revista Chilena de Historia Natural 67: 177-182.

González-Martínez, S.C., Bravo, F. 2001. Density and population structure of the natural regeneration of Scots pine (Pinus sylvestris L.) in the High Ebro Basin (Northern Spain). Annals of Forest Science 58: 277-288.

González, M.E., Lara, A., Urrutia, R., Bosnich, J. 2011. Cambio climático y su impacto potencial en la ocurrencia de incendios forestales en la zona centro-sur de Chile $\left(33^{\circ}\right.$ $42^{\circ} \mathrm{S}$ ). Bosque 32(3): 215-219.

Gómez, P., HAhn, S., SAN MARTín, J. 2009. Estructura y composición florística de un matorral bajo plantaciones de Pinus radiata D. Don en Chile central. Gayana Botánica 66(2): 256-268.

Iniguez, J.M., Swetnam, T.W., Yool, S.R. 2008. Topography affected landscape fire history patterns in southern Arizona, USA. Forest Ecology and Management 256: 295-303.

Keeley, J.E., Lubin, D., Fotheringham, C.J. 2003. Fire and grazing impacts on plant diversity and alien plant invasions in the southern Sierra Nevada. Ecological Applications 13: 13551374.

Le Quesne, C., Stahle, D.W., Cleaveland, M.K., Therrell, M.D., Aravena, J.C., Barichivich, J. 2006. Ancient Austrocedrus tree-ring chronologies used to reconstruct central Chile precipitation variability from A.D. 1200 to 2000 . Journal of Climate 19(22): 5731-5744.

McDonald, P.M., LaAcke, R.J. 1990. Pinus radiata D. Don. In: Burns, R.M., Honkala, B.H. (eds.), Silvics of North America: 1. Conifers, pp. 433-441, Forest Service, United 
States Department of Agriculture, Washington DC, USA.

Money, H., Kummerow, J. 1971. The comparative water economy of representative evergreen sclerophyll and drought deciduous shrubs of Chile. Botanical Gazette 132: 245252.

Montenegro, G., Avila, G., Schatte, P. 1983. Presence and development of lignotubers in shrubs of the Chilean matorral. Canadian Journal of Botany 61: 1804-1808.

Montenegro, G., Ginocchio, R., Segura, A., Keely, J., Gómez, M. 2004. Fire regimes and vegetation responses in two Mediterranean-climate regions. Revista Chilena de Historia Natural 77: 455-464.

Oliver, C., Larson, B. 1997. Forest Stand Dynamics. McGrawHill, Inc. 470 pp.

Pickett S.T., Collins, S.L., Armesto, J.J. 1987. Models, mechanisms and pathways of succession. The Botanical Review 53: 335-371.

Quintanilla, V. 2000. Influencia del fuego en el desequilibrio ecológico de la vegetación de la zona mediterránea de Chile: casos de estudio. Investigaciones Geográficas 34: 1-14.

Ramírez, C., Figueroa, F., Carrillo, R., Contreras, D. 1984.
Estudio fitosociológico de los estratos inferiores en un bosque de pino, Valdivia, Chile. Bosque 5(2): 65-81.

Richardson, D.M., Williams, P.A., Hobbs, R.J. 1994. Pine invasions in the southern hemisphere: Determinants of spread and invasibility. Journal of Biogeography 21: 511527.

Richardson, D.M., Pysek, P., Rejmánek, M., Barbour, M.G., PanetTA, F.D., West, C.J. 2000. Naturalization and invasion of alien plants: concepts and definitions. Diversity and Distributions 6: 93-107.

VIDAL, O.J., REIF, A. 2011. Effect of a tourist-ignited wildfire on Nothofagus pumilio forests at Torres del Paine biosphere reserve, Chile (Southern Patagonia). Bosque 32: 64-67.

Weiguo, S., Sha, C., Guangqi, L. 2008. Dynamics of leaf area index and canopy openness of three forest types in a warm temperate zone. Frontiers of Forestry in China 3: 416-421.

Zuloaga, F., Morrone, O., Belgrano, M. 2008. Catálogo de las plantas vasculares del Cono Sur (Argentina, Sur de Brasil, Chile, Paraguay, Uruguay). URL: http://www2.darwin. edu.ar/Proyectos/FloraArgentina/fa.htm

Recibido: 11.11.2015

Aceptado: 16.06.2017 